\title{
CORRELATION OF SECONDARY DENDRITE ARM SPACING WITH THE MECHANICAL BEHAVIOUR OF CAST 718 ALLOY
}

\author{
Natalia Soldevilla ${ }^{1}$, Oscar Caballero ${ }^{1}$ \\ ${ }^{1}$ Industria de Turbopropulsores; Parque Tecnológico 300, Zamudio, 48170, Spain
}

Keywords: Cast 718 alloy, Mechanical behaviour, Secondary dendrite arm spacing

\begin{abstract}
The aim of this study is to evaluate the influence of the microstructural parameters in the mechanical behaviour of casting nickel-based 718 alloy. This research has been focused on two of the key microstructural features, grain size and secondary dendrite arm spacing (SDAS), which are directly related to solidification conditions. Even though SDAS appears to be the most important parameter in castings, availability of studies is scarce. In this context, an own measurement methodology has been developed to analyze a wide range of samples from turbine components. Due to casting process sensitivity, significant variations in microstructure and related mechanical properties have been contemplated. As a result, it was found that both grain size and SDAS have an important role on the mechanical behavior and a correlation has been established with the studied properties (Tensile, LCF and Creep). From this correlation, resistance parameters and subsequent allowables can be predicted.
\end{abstract}

\section{Introduction}

Nickel-based superalloys are typically used for aircraft propulsion applications [1-2]. Turbine engines create harsh environments for materials due to the high operating temperatures and stress levels. The mechanical properties of cast nickel-based alloys can be optimized by tailoring both chemistry and microstructural features through casting process control.

The purpose of this study was to further evaluate the effect of various microstructural parameters that contribute to the control of the mechanical behaviour of one of the most important and frequently employed casting nickel-based 718 alloy. This research has been focused on studying two of the key microstructural features in the microstructure of a cast alloy, the grain size and the secondary dendrite arm spacing [3]. Even though secondary dendrite arm spacing appears to be the most important structural length parameter in castings, availability of studies on its quantification in nickel-based alloys is scarce. However, investigations on this feature have been widely conducted in light alloys [3-7].

The present investigation was carried out in order to elucidate and assess the influence of the casting process and resultant variations of microstructure on the mechanical behaviour response. The mechanical properties which have been studied in this work are: Tensile (Ultimate Tensile Strength, Yield Strength), Low Cycle Fatigue and Creep. The coarseness of the microstructure has been evaluated in terms of Grain Size (GS), Secondary Dendrite Arm Spacing (SDAS) and the phases evolved during solidification. 


\section{Experimental}

\section{$\underline{\text { Cast material }}$}

The material used for this research was cast using nickel-based 718 alloy, which was subsequently subjected to standard heat treatment as per AMS 5383. Both tests bars and components were considered to be of interest in order to analyse a wide range of dendritic solidified samples. Such significant variations in solidification conditions result in different coarseness of the microstructure and scatter in the related mechanical properties.

Specimens were machined from bearing flanges and vanes (i.e. Machined From Casting, MFC), Separately Cast Test Bars (i.e. SCTB), Integrally Cast Test Bars (i.e. ICTB) and cast plates, with the purpose of obtaining specimens suitable for tensile, low cycle fatigue and creep testing. Samples for microstructure assessment were excised from these testing specimens.

Samples were sectioned by a silicon carbide water-cooled cutoff wheel and mounted in a thermosetting resine. Then, sample preparation consisted of a standard series of wet grinding and polishing followed by chemical etching with Kalling's reagent 2.

In order to analyse the microstructure and measure the different microstructural features, the optical microscope Leica MZ 75 with Leica QWin image processing software and the scanning electron microscope, Philips XL 30 ESEM, were used. Since all tested material has been subjected to the standard ageing treatment which is supposed to be sufficiently developed for an optimized precipitation, it is important to remark that no TEM analysis has been carried out in order to assess the gamma double prime characteristics.

\section{$\underline{\text { SDAS measurement methodology }}$}

Secondary dendrite arm spacing can be defined as the distance between adjacent branches growing from the main dendritic spike or primary branch.

Several investigators have maintained that secondary dendrite arm spacing is a good index of solidification conditions in a casting. From the results of the SDAS measurement, information can be obtained regarding the rate of solidification of the material and therefore some indication of its strength. Increasing the cooling rate produces finer secondary dendrite arm spacing.

In order to quantify SDAS parameter, the following procedure was established. These parameter measurements were accomplished by randomly choosing five dendrites along each analysed sample, representing the calculated SDAS the average value as determined from at least ten measurements on each dendrite.

The measurements were made including only the branches which are termed "active", and have grown ahead of those that that do not grow much further from the primary branch. The reason for this way of measuring is that active branches are supposed to influence material properties to a much greater extent than the inactive ones [6]. A schematic representation of the performed measurements is given in Figure 1. 


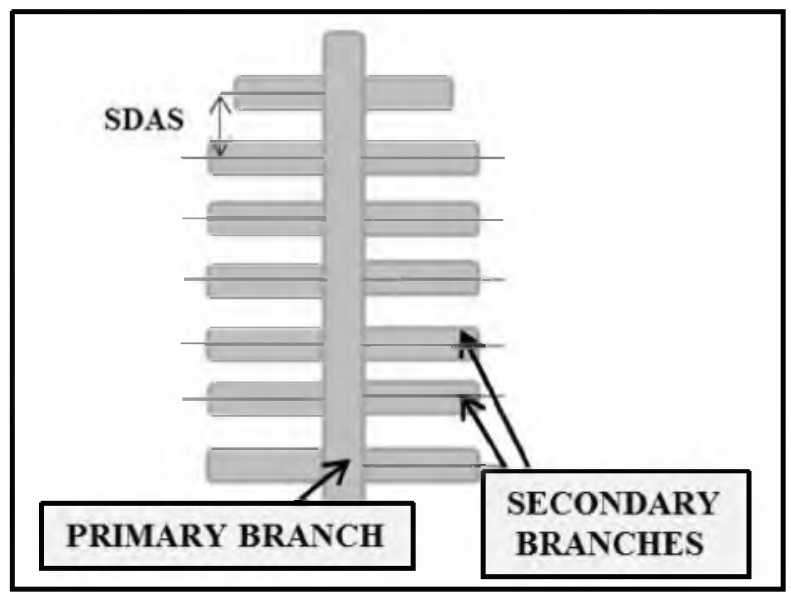

Figure 1. Schematic representation of SDAS

By means of this SDAS measurement methodology, the quantification of all the available samples for the correlation study, was carried out.

For instance, Figure 2 shows the ten SDAS measurements performed in one of the five randomly chosen dendrites. In this particular case, the metallographic sample was extracted from an ICTB.

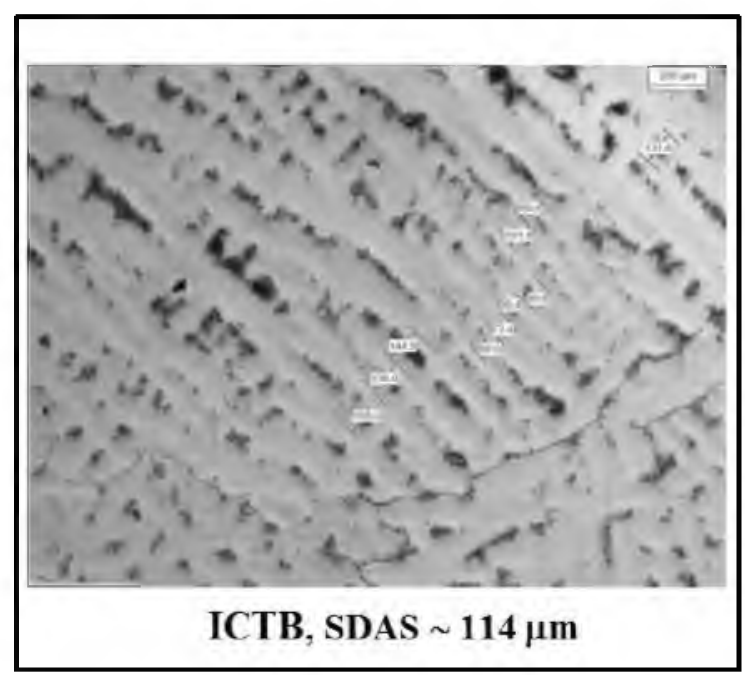

Figure 2. SDAS measurements along a dendrite

In order to complete the quantification, an average SDAS value was calculated considering the total number of measurements done per sample.

Later on, the standard deviation was determined in order to assess how much variation or dispersion from the mean value existed. As a result of these calculations, it was established that the standard deviation was low. Thus, the SDAS parameters were not spread out over a large range of values and the developed methodology was considered acceptable and representative. 


\section{Results and discussion}

\section{$\underline{\text { Microstructure }}$}

It is well known that two of the most important features of cast alloys are the grain size and the secondary dendrite arm spacing [3-7]. Even though SDAS appears to be the key structural length parameter in castings, availability of studies on its quantification in nickel-base superalloys is scarce. The aforementioned measurement methodology has been developed in order to quantify this parameter. Then, the influence of both grain size and SDAS has been studied and a correlation with the mechanical behaviour has been established.

Examining the microstructure of specimens from test bars (SCTB, ICTB), cast parts (MFC) and cast plates, it has been observed that there is a wide scatter in grain size, SDAS and significant variations of size and amount of the deleterious Laves phase. This brittle phase appears entirely in the interdendritic region, as this material is the last one to solidify.

Comparing the microstructure formations, it can be noticed that in the case of samples extracted from separately cast test bars the microstructure is finer, due to the faster solidification that takes place as a logical consequence of being cast in a separate mould. Furthermore, no remarkable differences between samples taken from MFC and ICTB can be detected since integrally cast test bars are poured as an integral part of a casting component. In these MFC and ICTB samples, the observed microstructure is coarse, and similar to the observed one in cast plates.

The different coarseness of microstructure as a function of the solidification conditions can be clearly seen in the following figures. Micrographs were taken with the optical microscope under the same magnification. Figure 3 shows a small secondary dendrite arm spacing in a sample extracted from SCTB.

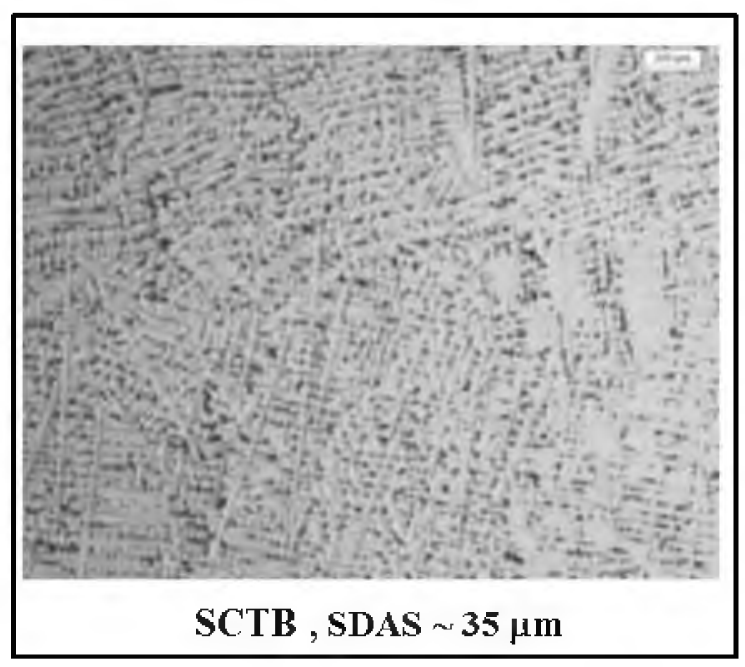

Figure 3. Microstructure in SCTB samples

In contrast, the microstructures of both MFC and ICTB samples are significantly coarser in terms of SDAS parameter. Typical microstructures are reported in Figure 4, being the SDAS values of a similar magnitude. 

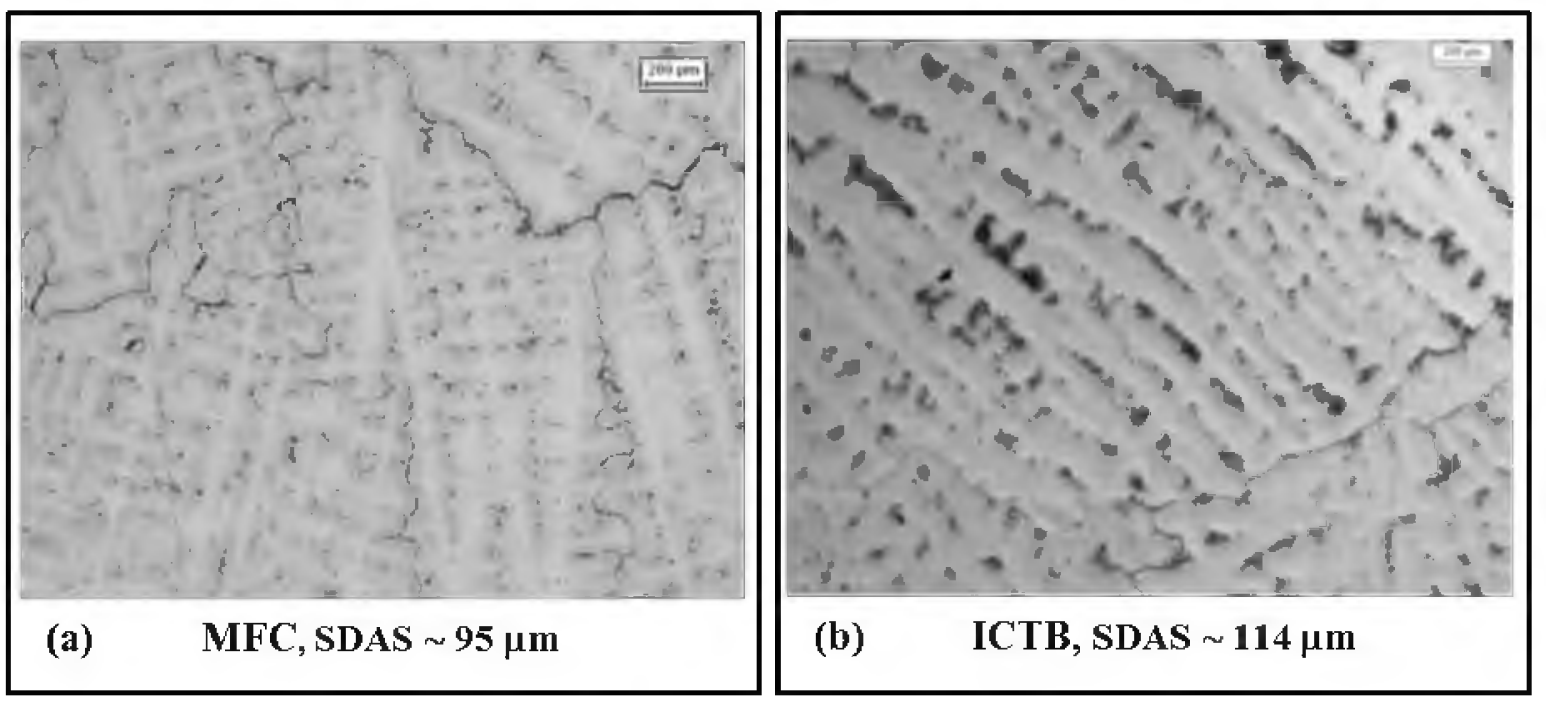

Figure 4. Microstructure in: (a) MFC and (b) ICTB samples

\section{$\underline{\text { Mechanical properties }}$}

While a lot of previous work in assessing the relation between the microstructure and the mechanical behaviour has been performed [8-10], there are still limited studies in designing microstructures with tailored mechanical resistance.

Since coarseness of microstructures are likely to be different due to microsegregation in casting components leading to local variations in mechanical properties, the purpose of this research was mainly to establish a correlation between microstructure and the mechanical behaviour of the cast 718 alloy. From this correlation, resistance parameters and subsequent design allowables could even be predicted in zones of the cast parts in which testing specimens cannot be machined due to geometry limitations.

In order to assess the influence of the observed microstructure variations on the mechanical behaviour response, the mechanical properties studied are: Tensile, Low Cycle Fatigue and Creep.

Tensile properties. The tensile properties which have been measured in this investigation are: Ultimate Tensile Strength (UTS) and Yield Strength (YS). The microstructural features that have been evaluated are grain size, secondary dendrite arm spacing and Laves phase. Since maximum operation temperature without significant degradation in properties is close to $600^{\circ} \mathrm{C}$, tensile properties of the material have been evaluated at this temperature.

The results of these tensile tests (from cylindrical specimens machined from SCTB, ICTB and MFC) and the calculated SDAS values are plotted in the graphs in Figure 5, for the analysed UTS and YS properties. Note that mechanical property data on the y axis have been normalized to maximum property value derived from the corresponding mechanical testing. All subsequent figures of this paper use the same normalizing technique. 


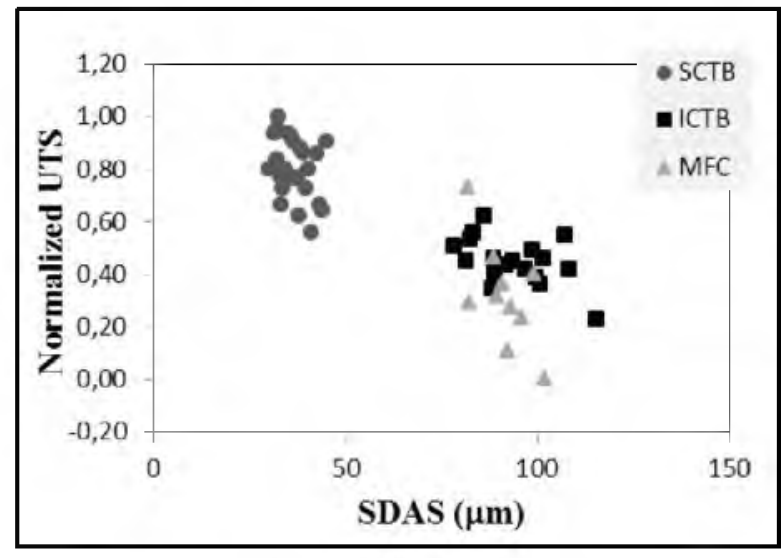

(a)

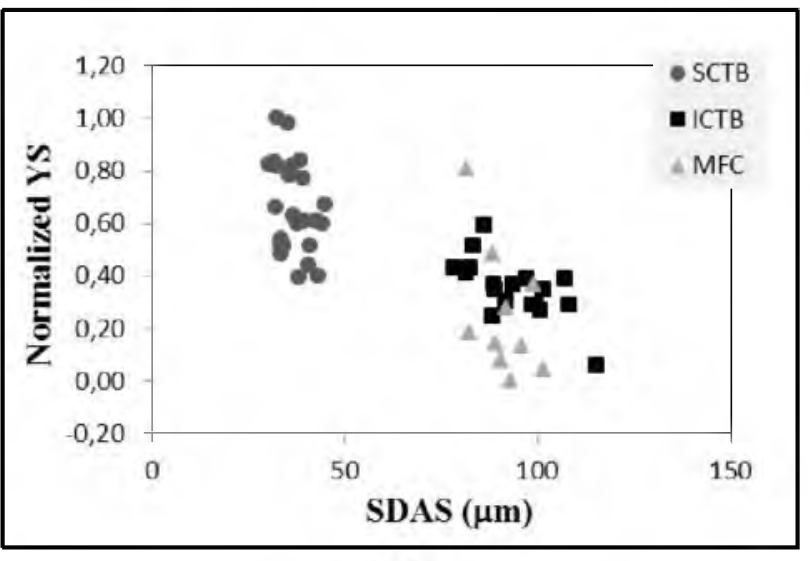

(b)

Figure 5. Correlation between: (a) SDAS and UTS (b) SDAS and YS

These graphs show clear variations in SDAS values, existing a correlation between this microstructural parameter and the resulting UTS and YS properties. SDAS parameter, strongly affected by the solidification conditions, is therefore directly related to test bar nature. Therefore, two distinguishable populations can be observed:

At high solidification rates the SDAS is smaller and the overall effect of obtaining such SDAS is an improvement in both ultimate tensile and yield strength. This population corresponds to test specimens extracted from SCTB, with average SDAS $\sim 37 \mu \mathrm{m}$.

On the contrary, slow solidification rates lead to larger SDAS values resulting in a reduction of tensile properties. This population consists of test specimens extracted from ICTB and MFC, with average SDAS $\sim 94 \mu \mathrm{m}$.

Despite the fact that these variations in cooling time lead to major differences in both GS (not reported herein) and SDAS impacting on the tensile performance of the cast bars, a careful analysis revealed that up to $3 \mathrm{~mm}$ grain size variation no property drop occurred.

Moreover, although Laves phase was present in most of the studied samples, no evidence of significant property drop was detected due to this phase.

In addition, it may be noted that the fracture surfaces were also examined in SEM. The fractograph of the samples did not reveal the presence of remarkable microshrinkage in any case, so this feature cannot be considered as a source of additional scatter in the data.

Therefore, taking into account that average grain size was identical for both populations and no noticeable segregations or shrinkage occurred, it can be concluded that the observed tensile behaviour can be solely attributed to the SDAS variations.

Low Cvcle Fatigue properties. Low Cycle Fatigue testing of this nickel-based alloy has been performed using axial loading, square waveform, $\mathrm{f}=0.25$ and $\mathrm{R}=0$ at $600^{\circ} \mathrm{C}$. In this particular case, available mechanical test specimens are excised from SCTB and cast plates exhibiting different thicknesses. Cylindrical specimens were machined from SCTB, whereas both geometries, cylindrical and flat test specimens, were addressed for the cast plates. 
Regarding microstructural characterization, the aforementioned considered features were once again studied following the same procedure accomplished for tensile test specimens. The analysis of the cylindrical specimens extracted from cast plates revealed that the microstructure was coarse and quite similar in terms of grain size and SDAS to the one previously observed in specimens taken from cast parts (MFC). On the contrary, it was clearly seen that the microstructure of the flat specimens was significantly finer due to the comparatively smaller plate thickness from which these specimens were machined. Nevertheless, it can also be noticed that dendritic pattern is not as fine as in specimens extracted from SCTB.

The results derived from cyclic loading tests performed at $600^{\circ} \mathrm{C}$ are shown in Figure 6. Since the outcome of the fatigue test is the relationship between two variables, the graph shows the normalized cyclic stress (Smax) against the logarithmic scale of cycles to failure $(\mathrm{N})$.
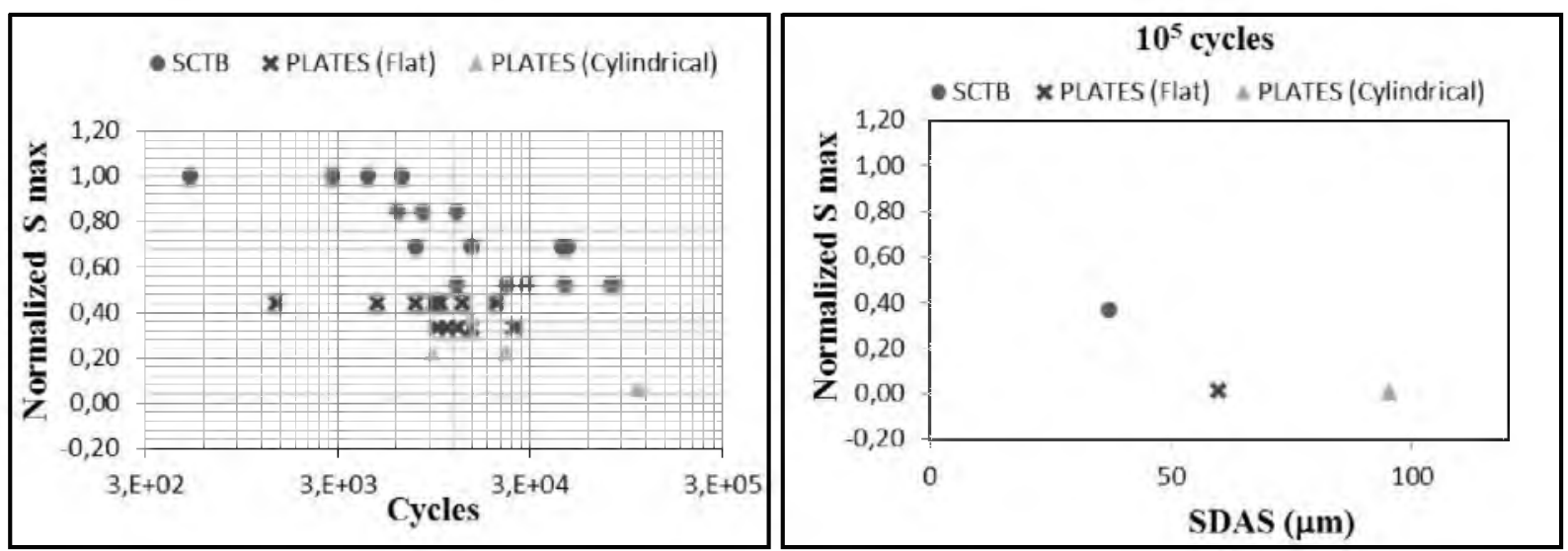

Figure 6. Smax-N diagram at $600^{\circ} \mathrm{C}$ (left), Smax at $10^{5}$ cycles vs SDAS (right)

Analyzing these results, it can be seen that material response to cyclic loading is quite good in specimens extracted from SCTB. In this sense, these results are in line with the previously observed behaviour of this kind of specimens when subjected to tensile testing. In contrast to this the fatigue results derived from cast plates have, however, been found to lie far below the SCTB results especially in cylindrical specimens.

In order to provide an easier comparison amongst the tested specimens and establish a direct relationship between the applied stress and SDAS, a fixed life of $10^{5}$ was considered as a reference point for comparison. The interpretation of the graphs shown in Figure 6 leads to the conclusion that, although three different populations are available in terms of SDAS parameter, a similar behaviour can be observed for all specimens taken from cast plates. In other words, it is interesting to observe that with the increase in SDAS parameter, a trend to decrease the fatigue resistance is confirmed. Nevertheless, an additional property drop occurred in flat specimens as it is shown in Smax-SDAS graph in Figure 6. It can be speculated that this lower fatigue resistance could be due to the edge effect as a consequence of the flat geometry shape.

Based on the aforementioned statements concerning grain size and Laves phase influence, it can be concluded that the secondary dendrite arm spacing associated with solidification conditions during the casting process, has also a key role in the material fatigue behaviour. Once again, no evidence of microshrinkage was observed in the studied specimens. 
Creep properties. Creep rupture experiments were also conducted to assess the influence of the grain size and SDAS parameters in the strength and lifetime of cast 718 alloy under quasi-static conditions at elevated temperatures: 600,650 and $700^{\circ} \mathrm{C}$. Test specimens were extracted from SCTB and cast plates exhibiting different thicknesses. The aforementioned microstructural features were once more studied.

Initially, this study was focused on studying two different data sets, specimens taken from SCTB and plates, tested at $650^{\circ} \mathrm{C}$. On the basis of the performed microstructure investigation and mechanical testing, it was concluded that higher rupture times were achieved in specimens machined from SCTB. The microstructure of these specimens, as previously reported for tensile and fatigue properties, showed a much smaller SDAS value $(\sim 40 \mu \mathrm{m})$ when compared to the one observed in specimens extracted from cast plates $(\sim 80 \mu \mathrm{m})$. In spite of this different SDAS, no meaningful variations were observed in terms of grain size $(\triangle \mathrm{GS} \sim 1 \mathrm{~mm})$. Once normalized, the results derived from creep rupture tests performed at $650^{\circ} \mathrm{C}$ are shown in Figure 7 , where significant creep strength reductions are evident for plate specimens.

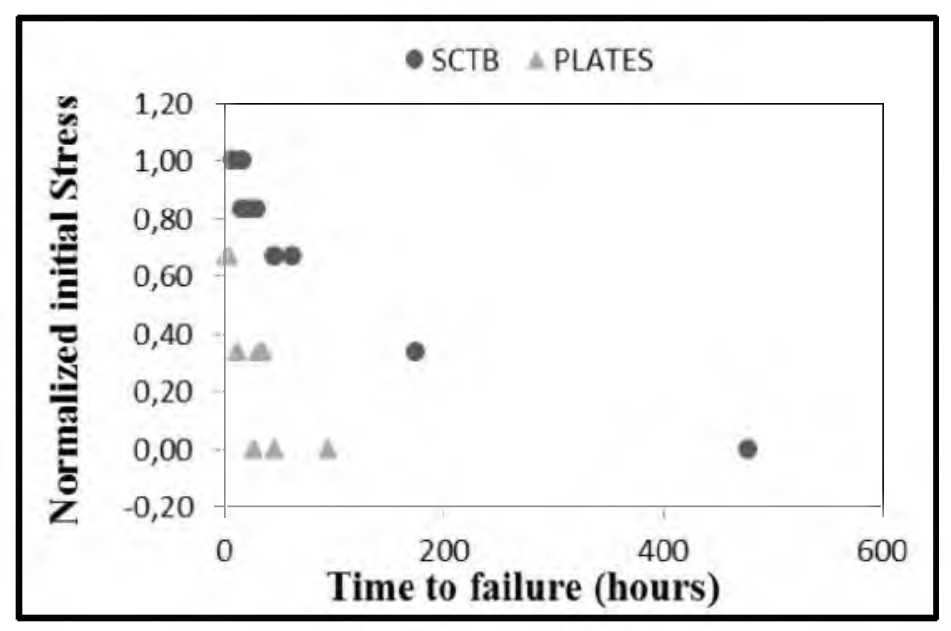

Figure 7. Predominant influence of SDAS

Later on, a complementary investigation was performed in cast plates. The aim of this other study was to analyse creep test results of material presenting now a similar SDAS parameter $(\sim 80 \mu \mathrm{m})$, but noticeable grain size differences $(\Delta \mathrm{GS} \sim 1.5 \mathrm{~mm})$.

Analysing the results at $600^{\circ} \mathrm{C}$, it can be noticed that Fine Grain size (FG) provides slightly better creep behaviour than the Coarse Grain size (CG). Nevertheless, when tests results at $700^{\circ} \mathrm{C}$ were compared, both data sets exhibit similar creep resistance. This might be a consequence of gamma double prime phase coarsening due to testing at $700^{\circ} \mathrm{C}$, which is above stability temperature of this alloy. Figure 8 shows the normalized results derived from testing these specimens at 600 and $700^{\circ} \mathrm{C}$. 


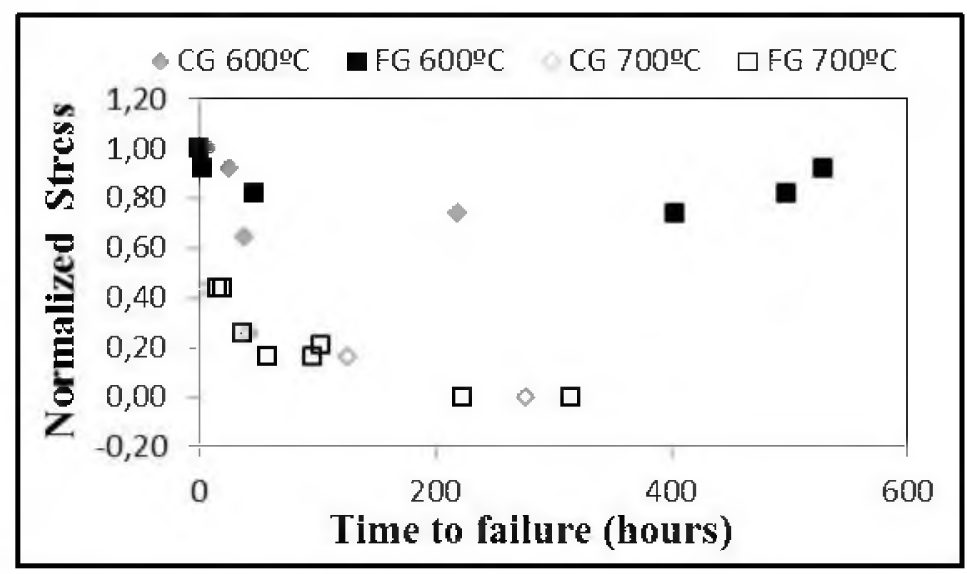

Figure 8. Influence of GS in creep behaviour

Finally, the creep rupture properties of specimens extracted from the same cast plate were studied in order to assess the internal scatter observed in the microstructure. Characterization of the features was completed to understand the observed differences regarding creep performance.

As a result of this investigation, it was deduced that specimens machined from material in outer plate locations showed a substantial improvement in rupture life when compared to the specimens extracted from inner locations. The observed behaviour can be attributed to the microstructural variations in both grain size and SDAS parameters $(\Delta \mathrm{GS} \sim 1 \mathrm{~mm}, \Delta \mathrm{SDAS} \sim 15$ $\mu \mathrm{m})$. These differences in sample location within the plate lead to local cooling time variations which affect the microstructure and therefore the mechanical behaviour of the alloy.

Nevertheless, for the same initial stress intensity, the time to failure reduction at $700^{\circ} \mathrm{C}$ is not as meaningful as for specimens tested at $600^{\circ} \mathrm{C}$. As mentioned before, the more uniform material performance at $700^{\circ} \mathrm{C}$ might be a result of the hardening phase degradation being grain size and SDAS not the only ruling factors. Figure 9 shows creep rupture results for outer location specimens (Fine Microstructure, FM) and inner location (Coarse Microstructure, CM) at both test temperatures.

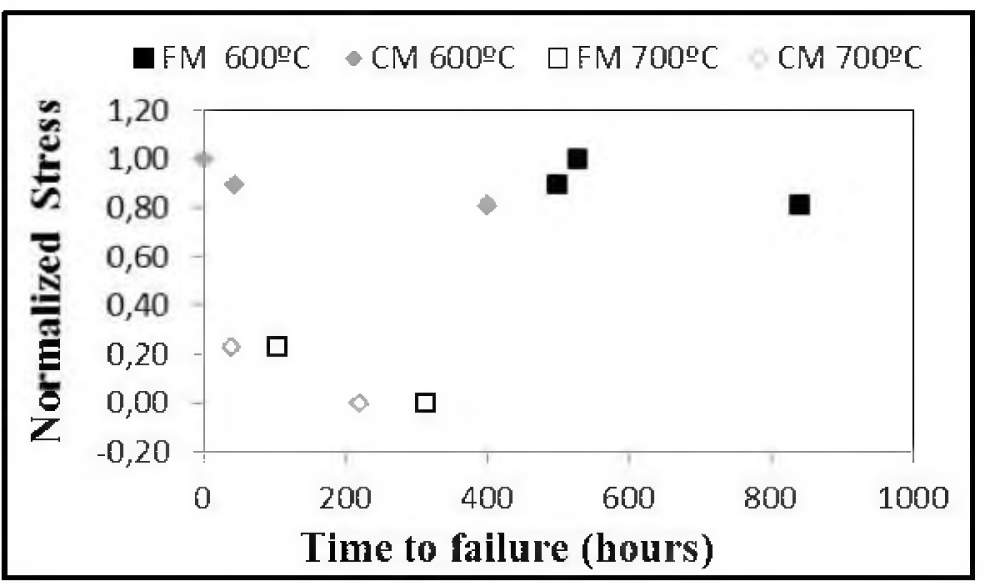

Figure 9. Combined effect of GS and SDAS 
Finally, these experimental data were classified into three groups according to their grain size and SDAS parameter combination. These groups are listed below:

Group A includes specimens showing coarse GS and large SDAS. These specimens were extracted from plates.

Group B includes specimens with fine GS and intermediate SDAS. Although specimens were also excised from plates, the outer location within the plate is responsible for the finer microstructure when compared to specimens in Group A.

Group C is characterized by fine GS and small SDAS. Specimens were machined from SCTBs.

Once this classification was carried out, the Larson-Miller plot in Figure 10 was constructed in order to evaluate the influence of these microstructural parameters in the material creep performance.

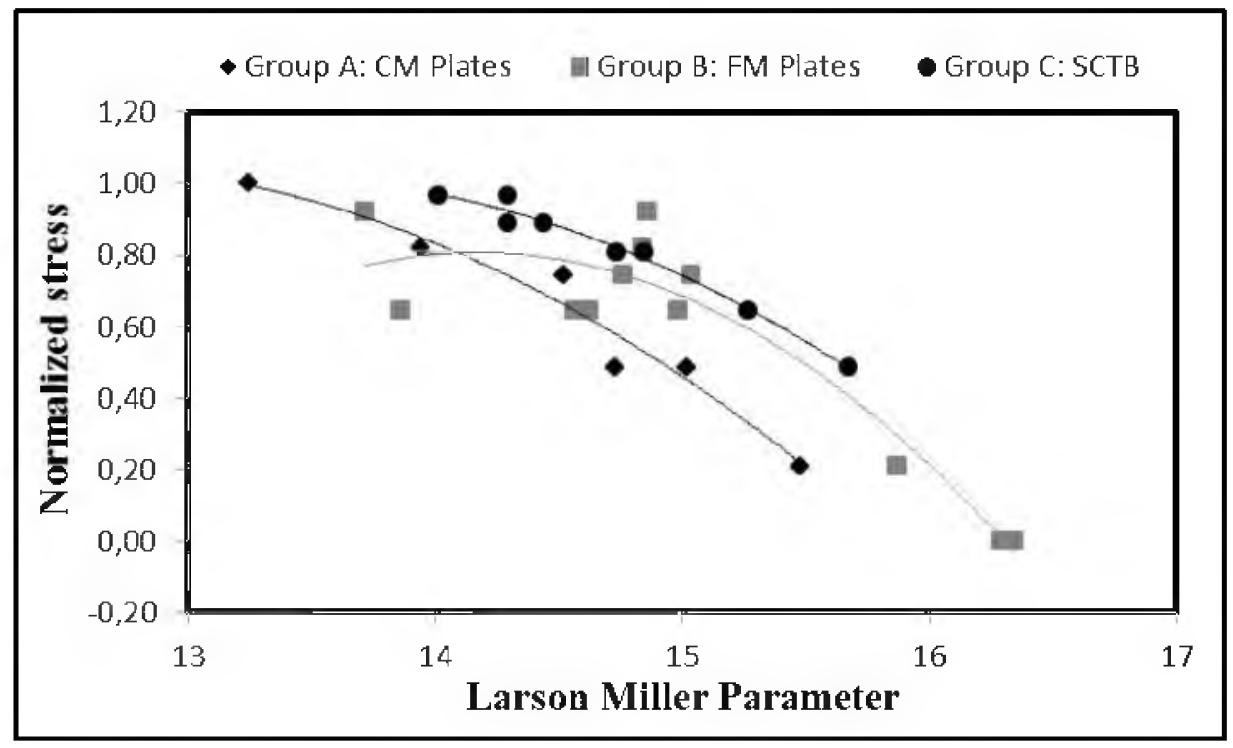

Figure 10. Larson-Miller plot of stress-rupture capability

The plot reveals a clear trend: the coarser the microstructure (coarser GS and larger SDAS) the more left-shifted curve and therefore, the lower rupture time according to Larson-Miller equation:

$$
\mathrm{LMP}=\mathrm{T}\left(\mathrm{C}+\log \mathrm{t}_{\mathrm{r}}\right)
$$

where LMP is known as the Larson Miller Parameter, $\mathrm{C}$ is a material constant, $\mathrm{T}$ represents the temperature and $t_{\mathrm{r}}$ represents the rupture time. 


\section{Conclusions}

Based on the current investigation concerning the microstructural characterization of the cast nickel-based 718 alloy and its mechanical behaviour, it can be concluded that the secondary dendrite arm spacing (SDAS), associated with the solidification rate during the casting process, has a key role on the resulting mechanical behaviour response.

Specimens corresponding to different cooling rates have been subjected to mechanical testing and subsequent microstructural analysis. Different coarseness of microstructures as a function of the solidification conditions have been observed, being the microstructure of specimens extracted from SCTB significantly finer when compared with the other studied ones. The higher the cooling rate the finer the microstructure.

With regards to tensile behaviour, the overall effect of obtaining a small SDAS is an improvement in both ultimate tensile and yield strength.

Concerning fatigue test results, although a finer microstructure with smaller SDAS is also beneficial, a clear correlation between this parameter and the stress allowables cannot be established since two types of fatigue testing specimens have been considered. This fact, together with the well-known influence of many factors in the fatigue response, contributes to a greater data scatter.

A smaller SDAS has resulted in substantial improvement of material behaviour under creep conditions.

Even though it was found that variations in both grain size and SDAS influence the mechanical response, the experimental results suggest that SDAS parameter plays a key role over grain size strongly affecting the mechanical behaviour of this alloy. From the observed relationships a correlation with mechanical properties could also be established.

\section{References}

1. R. P. Jewett and J. A. Halchak, "The use of alloy 718 in the space shuttle main engine," in E. A. Loria (Ed.), Proceedings of the Conference on Superalloys 718, 625 and Various Derivatives, TMS, Warrandale, PA, (1991) 749-760.

2. D. Fournier and A. Pineau, "Low cycle fatigue behaviour of Inconel 718 at $298 \mathrm{~K}$ and $823 \mathrm{~K}$," Metallurgical Transactions A, 8A, (1977), 1095-1105.

3. M. Easton, C. Davidson, and D. StJohn, "Grain morphology of as-cast wrought aluminium alloys," Proceedings of the $12^{\text {th }}$ International Conference on Aluminium Alloys, The Japan Institute of Light Metals, Yokohama, (2010), 173-178.

4. S. Seifeddine, T. Sjögren, and I. L. Svensson, "Variations in microstructure and mechanical properties of cast aluminium EN AC 43100 alloy," Metallurgical Science \& Technology Journal, 25, $\mathrm{N}^{\mathrm{o}}$ 1, (published by Teksid Aluminum, 2007), 12-22. 
5. M. Merlin and G. L. Garagnani, "Mechanical and microstructural characterisation of A356 castings realised with full and empty cores," Metallurgical Science \& Technology Journal, 27, $\mathrm{N}^{\mathrm{o}} 1$, (published by Teksid Aluminum, 2009), 21-30.

6. R. N. Grugel, "Secondary and tertiary dendrite arm spacing relationships in directionally solidified Al-Si alloys," Journal of Materials Science, 28, (1993), 677-683.

7. D. M. Rosa, J. E. Spinelli, and A. Garcia, "Tertiary dendrite arm spacing during downward transient solidification of Al-Cu and Al-Si alloys," Materials Letters, 60, (2006), 1871-1874.

8. L. Kyuhong, Y. N. Kwon, and S. Lee, "Correlation of microstructure with mechanical properties and fracture toughness of A356 aluminium alloys fabricated by low-pressurecasting, rheo-casting, and casting-forging processes," Engineering Fracture Mechanics, 75, (2008), 4200-4216.

9. C. Przybyla, R et al., "Microstructure-sensitive modelling of high cycle fatigue," International Journal of Fatigue, 32, (2010), 512-525.

10. S. Zhao, X. Xie, G. D. Smith, and S. J. Patel, "Microstructural stability and mechanical properties of a new nickel-based superalloy," Materials Science and Engineering, A, 355, issues 1-2, (2003), 96-105. 